\title{
Genomic analysis of a nontoxigenic, invasive Corynebacterium diphtheriae strain from Brazil
}

\author{
Fernando Encinas ${ }^{1}$, Michel A Marin ${ }^{1 /+}$, Juliana N Ramos ${ }^{1,2,3}$, Verônica V Vieira', \\ Ana Luiza Mattos-Guaraldi², Ana Carolina P Vicente
}

1Fundação Oswaldo Cruz, Instituto Oswaldo Cruz, Laboratório de Genética Molecular de Microrganismos, Rio de Janeiro, RJ, Brasil
${ }^{2}$ Universidade do Estado do Rio de Janeiro, Faculdade de Medicina, Laboratório de Difteria e Corinebactérias de Importância Clínica,
Rio de Janeiro, RJ, Brasil ${ }^{3}$ Fundação Oswaldo Cruz, Instituto Nacional de Controle de Qualidade em Saúde, Rio de Janeiro, RJ, Brasil

We report the complete genome sequence and analysis of an invasive Corynebacterium diphtheriae strain that caused endocarditis in Rio de Janeiro, Brazil. It was selected for sequencing on the basis of the current relevance of nontoxigenic strains for public health. The genomic information was explored in the context of diversity, plasticity and genetic relatedness with other contemporary strains.

Key words: Corynebacterium diphtheriae - ST-171 - nontoxigenic

Corynebacterium diphtheriae is the causative agent of diphtheria, a toxaemic disease that is controlled through immunisation programs. Infections caused by nontoxigenic strains of $C$. diphtheriae are not preventable by vaccination even though may cause severe invasive infections such as endocarditis and septic arthritis (Zasada et al. 2010, Peixoto et al. 2014). Outbreaks appear to be followed by periods of increasing genomic diversity (Mokrousov 2009) therefore, there is a need to explore the genomic information of these strains in terms of diversity and plasticity related to potential virulence determinants. In such a context, we describe the sequencing, annotation and analysis process of $\mathrm{HC} 07$, a nontoxigenic, invasive $C$. diphtheriae strain from Rio de Janeiro (RJ), Brazil.

$\mathrm{HC} 07$ was isolated in 2013 from the blood of a patient with endocarditis and belongs to biotype gravis. Identification and biotype determination were performed by conventional microbiological methods and the API Coryne System v.3.0 (bioMérieux, France) with the apiweb ${ }^{\mathrm{TM}}$ web decoding system. HC07 was characterised as nontoxigenic by the modified Elek test at the Centers for Disease Control and Prevention (USA) and the Vero cell cytotoxicity assay. The multilocus sequence typing (MLST) scheme using seven housekeeping genes (atp $\mathrm{A}, \operatorname{dn} a \mathrm{E}, \operatorname{dn} a \mathrm{~K}$, fus $\mathrm{A}, l e u \mathrm{~A}, o d h \mathrm{~A}$ and $r p o \mathrm{~B}$ ) assigned the profile 2-2-3619-3-3-6 that corresponds to ST-171. The HC07 genome was sequenced using a Nextera paired-end library in an Illumina HiSeq 2500 sequencer (Oswaldo Cruz Foundation high-throughput sequencing platform). Assemblies were generated with the A5-miseq pipeline v.20140604 (arxiv. org/abs/1401.5130) followed by a gene prediction and an-

doi: 10.1590/0074-02760150250

Financial support: FNS/SVS (25030001256201331), PROEP, FAPERJ

- fellowships: CAPES-PNPD, CNPq

+ Corresponding author: mfabanto@ioc.fiocruz.br

Received 3 July 2015

Accepted 23 July 2015 notation process using the National Center for Biotechnology Information Prokaryotic Genomes Automatic Annotation Pipeline (Angiuoli et al. 2008). The shotgun project has been deposited at DDBJ/EMBL/GenBank under the accession JRUZ00000000 and version JRUZ00000000.1. The sequencing process rendered $16,360.560$ reads $(100$ bp mean length) representing a 620x coverage, assembled into 70 contigs. Basic genomic features are as follows: total size, 2,491.635 bp, GC content, $53.53 \%$, number of genes, 2,333, rRNAs, 6 (5S, 16S, 23S), and tRNAs, 51.

Phylogenetic reconstruction considering concatenated MLST genes $(18,218 \mathrm{bp}$ ) showed that HC07 (gravis/ isolated in 2013/ST-171) clusters with HC03 (mitis/isolated in 2000/ST-171) and HC04 (mitis/isolated in 2003/ ST-128) (data not shown), these three strains are nontoxigenic and were isolated in RJ from patients with endocarditis. Moreover, a whole-genome based phylogeny also showed their close relationship (Fig. 1) supporting thus a previous report that biovar classification does not correlate with $C$. diphtheriae phylogeny (Sangal et al. 2014). All genomes were aligned using Progressive Mauve (Darling et al. 2010) and a neighbour-joining phylogenetic tree was constructed in SeaView (Gouy et al. 2010) on 16 complete $C$. diphtheriae genomes. Potential virulence factors in $\mathrm{HC} 07$ were mined using the Virulence Factors Database (mgc.ac.cn/VFs/). Two gene clusters were found: SpaA (NG01 07265-NG01 07280) and SpaH (NG01_09430; NG01_110470-NG01_11485) that encode a complete set of pilus proteins and their respective cognate sortases. Prophage and genomic island (GI) predictions were performed using the Phispy algorithm (Akhter et al. 2012) and the IslandViewer web server (pathogenomics.sfu.ca/islandviewer). HC07 harbours a putative prophage (NG01 04220-NG01 04530) that neither corresponds to the corynephage which usually carries the diphtheric toxin gene, nor to the one identified in HC03. Furthermore, 24 GIs were predicted and their genomic content is mostly represented by hypothetical proteins. Together, these results represent an overview of the diversity and plasticity of a nontoxigenic $C$. diphtheriae genome in terms of accessory gene content (Fig. 2). 


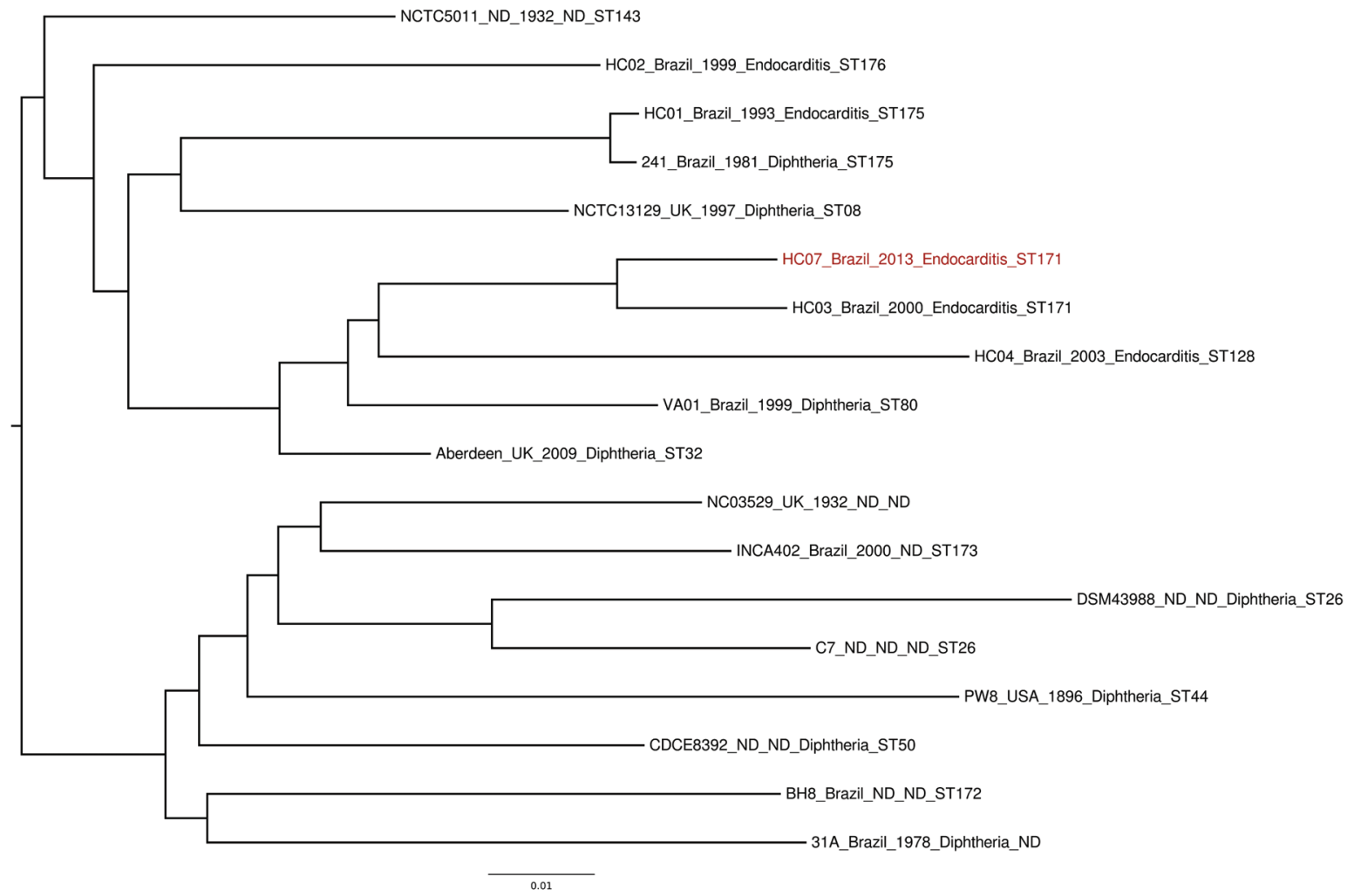

Fig. 1: phylogenomic tree including HC07 and other toxigenic/nontoxigenic Corynebacterium diphtheriae. Each genome is labelled by isolate name, country, year, disease and sequence type. ND: not determined.

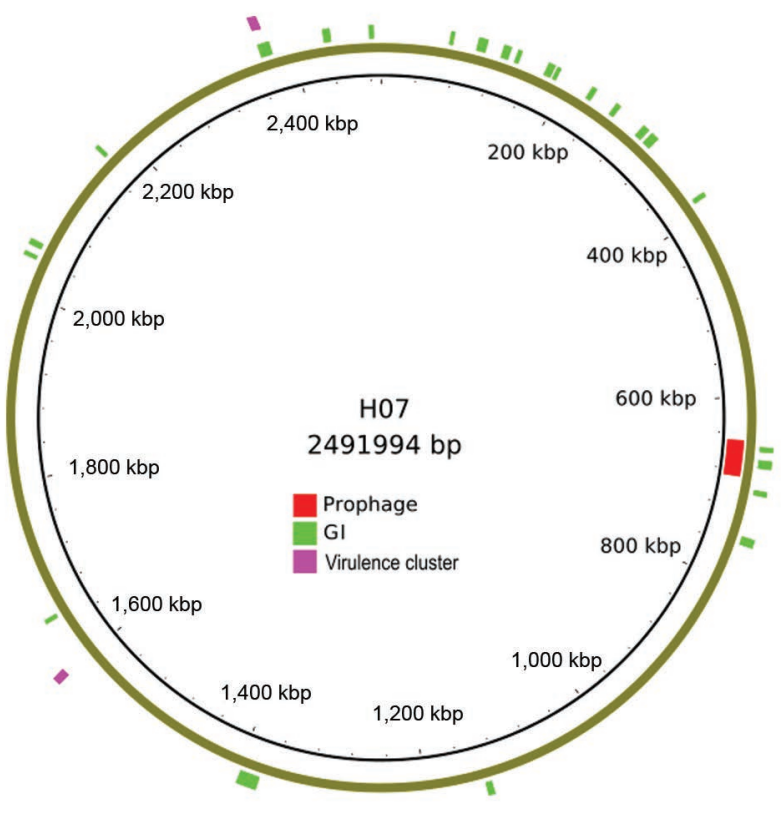

Fig. 2: circular representation of $\mathrm{HC} 07$ genome. Prophage, genomic islands (GIs) and virulence clusters are showed in red, green and purple, respectively.
Genomic analyses, including phylogenetic reconstructions, showed that $C$. diphtheriae $\mathrm{HC} 07$ clusters with other nontoxigenic endocarditis causative strains isolated in the same region more than a decade apart. This suggests the existence and persistence of a lineage that has been evolving and recurring in RJ. Particularly, $\mathrm{HC} 07$ harbours a set of potential virulence factors, a prophage and various GIs that characterise its diversity and plasticity that should be explored deeply in order to understand $C$. diphtheriae evolution.

\section{REFERENCES}

Akhter S, Aziz RK, Edwards RA 2012. PhiSpy: a novel algorithm for finding prophages in bacterial genomes that combines similarity and composition-based strategies. Nucleic Acids Res 40: e126.

Angiuoli SV, Gussman A, Klimke W, Cochrane G, Field D, Garrity G, Kodira CD, Kyrpides N, Madupu R, Markowitz V, Tatusova T, Thomson N, White O 2008. Toward an online repository of Standard Operating Procedures (SOPs) for (meta)genomic annotation. OMICS 12: 137-141.

Darling AE, Mau B, Perna NT 2010. progressiveMauve: multiple genome alignment with gene gain, loss and rearrangement. PLoS ONE 5: e11147.

Gouy M, Guindon S, Gascuel O 2010. SeaView version 4: a multiplatform graphical user interface for sequence alignment and phylogenetic tree building. Mol Biol Evol 27: 221-224. 
Mokrousov I 2009. Corynebacterium diphtheriae: genome diversity, population structure and genotyping perspectives. Infect Genet Evol 9: 1-15.

Peixoto RS, Pereira GA, dos Santos LS, Rocha-de-Souza CM, Gomes DL, dos Santos CS, Werneck LM, Dias AA, Hirata Jr R, Nagao PE, Mattos-Guaraldi AL 2014. Invasion of endothelial cells and arthritogenic potential of endocarditis-associated Corynebacterium diphtheriae. Microbiology 160: 537-546.
Sangal V, Burkovski A, Hunt AC, Edwards B, Blom J, Hoskisson PA 2014. A lack of genetic basis for biovar differentiation in clinically important Corynebacterium diphtheriae from whole genome sequencing. Infect Genet Evol 21: 54-57.

Zasada AA, Baczewska-Rej M, Wardak S 2010. An increase in nontoxigenic Corynebacterium diphtheriae infections in Poland-molecular epidemiology and antimicrobial susceptibility of strains isolated from past outbreaks and those currently circulating in Poland. Int J Infect Dis 14: e907-e912. 